\title{
Associations for BCO2, PCSK9, and TR1B1 Polymorphism and Lifestyle Factors with Ischemic Stroke: A Nested Case-Control Study
}

\author{
Tian-Yu Zhao ${ }^{1,2}$, Zheng $\mathrm{Li}^{2}$, Song Lei ${ }^{1,2}$, Liu Huang ${ }^{2}$, and Lei Yang ${ }^{2}$ \\ ${ }^{1}$ Medical School, Shihezi University, Shihezi, Xinjiang; \\ ${ }^{2}$ Medical School, Hangzhou Normal University, Hangzhou, Zhejiang, China.
}

\begin{abstract}
Purpose: To investigate associations for polymorphisms in $\beta$-carotene 9',10'-oxygenase (BCO2, rs10431036 and rs11214109), proprotein convertase subtilisin kexin type 9 (PCSK9, rs11583680), and tribbles pseudokinase 1 (TRIB1, rs17321515 and rs2954029), as well as lifestyle factors, with ischemic stroke (IS).

Materials and Methods: This nested case-control study included 161 patients with IS and 483 matched control individuals. We collected medical reports, lifestyle details, and blood samples from individuals and used the PCR-ligase detection reaction method to genotype single nucleotide polymorphisms (SNPs).

Results: The GA+AA genotype of rs10431036 ( $p<0.001)$ and rs17321515 ( $p=0.003)$, the CT+TT genotype of rs11214109 $(p=0.005)$, and the TA+AA genotype of rs2954029 ( $p=0.006)$ in dominant models increased the risk of IS. In additive models, the GG genotype of rs17321515 ( $p=0.005)$ and the TT genotype of rs2954029 ( $p=0.008)$ increased the risk of IS. Adequate intake of fruits/vegetables reduced the risk of IS ( $p=0.005)$. Although there was no interaction between genes and fruits/vegetables, people with inadequate intake of fruits/vegetables who carried a risk genotype had a higher risk of IS than those only having inadequate fruits/ vegetables intake or those only carrying a risk genotype. Also, the haplotypes AC, AT, and GT (comprising rs10431036 and rs11214109) and GT (comprising rs2954029 and rs17321515) were found to be associated with an increased risk of IS $(p<0.05)$.

Conclusion: Polymorphisms in $B C O 2$ and TRIB1 and fruits/vegetables intake were associated with IS. These results provide the theoretical basis for gene screening to prevent chronic cerebrovascular diseases.
\end{abstract}

Key Words: BCO2, PCSK9, TR1B1, polymorphism, ischemic stroke

\section{INTRODUCTION}

Stroke, as a heterogeneous syndrome, is one of the leading causes of mortality and morbidity, with astronomical financial repercussions for health systems worldwide. According to the World Health Organization, 15 million people suffer strokes

\footnotetext{
Received: March 18, 2019 Revised: April 22, 2019

Accepted: May 9, 2019

Corresponding author: Lei Yang, PhD, Medical School, Hangzhou Normal University, 16 Xuelin Rd, Hangzhou, Zhejiang, China.

Tel: 86-571-28865010, Fax: 86-571-28865000, E-mail: yanglei62@hznu.edu.cn

-The authors have no potential conflicts of interest to disclose.

(C) Copyright: Yonsei University College of Medicine 2019

This is an Open Access article distributed under the terms of the Creative Commons Attribution Non-Commercial License (https://creativecommons.org/licenses/ by-nc/4.0) which permits unrestricted non-commercial use, distribution, and reproduction in any medium, provided the original work is properly cited.
}

worldwide each year. Of these, more than 6 million die and another 5 million are permanently disabled. ${ }^{1}$ Epidemiological studies have indicated that about $70 \%$ of cases worldwide are ischemic strokes (IS), which are featured by disruption of cerebral blood flow and a hypoxia in the affected area. ${ }^{2}$

IS is caused by many factors, such as diabetes mellitus, obesity, dyslipidemia, and atherosclerosis..$^{3-5}$ It has a strong genetic basis, demonstrated by family associations and degrees of genetic influence as high as $40-50 \%$. Genetic studies have shown that a few specific gene variants of lipid metabolism affect the pathogenesis of IS. ${ }^{6-10}$ Proprotein convertase subtilisin/kexin type 9 (PCSK9) plays a major part in type 2 diabetes and atherogenesis development, which ultimately leads to IS. ${ }^{11,12} \beta$-carotene 9',10'-oxygenase (BCO2) and tribbles pseudokinase 1 (TRIB1) are important to maintaining normal lipid and cholesterol homeostasis, ${ }^{13,14}$ reducing the occurrence of cardiovascular and 
cerebrovascular diseases. In addition, lifestyle factors, such as fruits and vegetables intake, have been suggested as influencing factors for IS. ${ }^{15-17}$

Therefore, we sought to investigate associations for five single nucleotide polymorphisms (SNPs) in BCO2, PCSK9, and TRIB1 and for lifestyle factors (fruits/vegetables intake) with IS in southern China by a community-based nested case-control study.

\section{MATERIALS AND METHODS}

\section{Subjects}

A total of 2323 people who underwent physical examinations at the community health service center and had dyslipidemia from April 2013 to July 2013 were randomly selected from four townships under the jurisdiction of a certain district of Ningbo, Zhejiang Province as a survey point using cluster random sampling. All subjects were unrelated and $>40$ years of age. Tumors, aneurysms, patients after surgery or trauma, infections, subarachnoid hemorrhage, and women during pregnancy and baby nursing period were excluded. We gather all the subjects' life behavior information and blood samples. In order to understand the incidence of IS among the subject, we paid a visit in August 2016. The case group comprised 161 patients diagnosed with IS between April 2013 and August 2016. A total of 483 subjects were matched 1:3 as controls according to age and gender. All participants joined this study with informed consents, which got the permission by the Medical Ethics Committee of Hangzhou Normal University (No. 2013020).

\section{Diagnostic criteria}

Dyslipidemia was defined according to the Guidelines for the Prevention and Treatment of Diabetes in Chinese Adults prepared by the Joint Committee of the Chinese Association for the Prevention and Treatment of Dyslipidemia. Total cholesterol $(\mathrm{TC})>5.18 \mathrm{mmol} / \mathrm{L}$, triglycerides $(\mathrm{TG})>1.70 \mathrm{mmol} / \mathrm{L}$, high-density lipoprotein cholesterol (HDL-C) $<1.04 \mathrm{mmol} / \mathrm{L}$, and low-density lipoprotein cholesterol (LDL-C) $>3.37 \mathrm{mmol} / \mathrm{L}$ represent abnormal values. One or more of the four blood lipid indicators above were deemed indicative of a diagnosis of dyslipidemia. Subjects diagnosed with dyslipidemia and taking hypolipidemic drugs were also considered under the dyslipidemia category. Diagnosis of IS was based on the diagnostic criteria issued by the Society of Neurology, Chinese Medical Association in $2014 .{ }^{18}$ Patients were diagnosed as having IS with clinical manifestation and brain MRI and/or head CT.

\section{Epidemiological investigation}

The field epidemiological survey mainly consisted of fundamental demographic criteria such as age, sex, occupation, and education level, as well as information on lifestyle such as smoking, drinking, the intake of fruits/vegetables. The intake of fruits/vegetables and fruits survey employed a semi-quantita- tive questionnaire: average daily intake of fruits/vegetables less than $300 \mathrm{~g}$ was defined as "inadequate fruits/vegetables intake," and average daily fruits/vegetables intake more than $300 \mathrm{~g}$ was defined as "adequate fruits/vegetables intake." Anthropometric data, including body mass index (BMI), waist circumference, systolic blood pressure (SBP), diastolic blood pressure (DBP), and HDL-C and LDL-C levels, were evaluated by professional medical examination according to standard protocols.

\section{SNP selection and genotyping}

Five SNPs in BCO2, TRIB1, and PCSK9 were selected using the HapMap website (http://www.hapmap.org) and the Haploview 4.2 software (Broad Institute, Cambridge, MA, USA). The SNPs included rs10431036 and rs11214109 in BCO2, and rs17321515 and rs2954029 in TRIB1, rs11583680 in PCSK9. The minor allele frequencies (MAFs) of these five SNPs were greater than $5 \%$, and the linkage disequilibrium (LD) was $r^{2}>0.8$.

A refrigerator at $-80^{\circ} \mathrm{C}$ was selected to store $5 \mathrm{~mL}$ of whole blood from each fasted individual, which was anticoagulated with EDTA. DNA was extracted using Tiangen Blood Genomic DNA extraction kits (Tiangen Biotech, Beijing, China) and sent to Shanghai Jierui Biological Engineering Co., Ltd. (Shanghai, China), for genotyping analysis using the PCR-ligase detection reaction method (Generay Biotech Company, Shanghai, China). For quality control, we randomly chose $10 \%$ of samples for regenotyping, and the concordance rates were 96.8\% (rs10431036), 97.7\% (rs11214109), 98.9\% (rs17321515), and 99.2\% (rs2954029).

\section{Statistical analysis}

Statistical analysis was completed using SPSS 24.0 software (IBM Corp., Armonk, NY, USA). Demographic characteristics and the SNP genotypes of genes were evaluated using Student's t-test, $\chi^{2}$ test, Fisher's exact test (for categorical variables), and Wilcoxon's rank sum test (for continuous variables). The $\chi^{2}$ test was used to test for Hardy-Weinberg equilibrium (HWE). Allelic frequencies were analyzed using Fisher's exact test to generate the reported $p$ values. Logistic regression analysis was used to determine the odds ratio (OR) of the associations between genetic model and lifestyle factors and IS risk. Using Microsoft Excel according to Knol, et al., ${ }^{19}$ we identified relative excess risk due to interaction (RERI), ORs, and 95\% confidence intervals (CIs). The associations between gene haplotypes and the risk of IS were calculated using the R language package "haplo.stats" (R Foundation for Statistical Computing, Vienna, Austria). In all analyses, differences were deemed significant if $p<0.05$.

\section{RESULTS}

\section{Basic characteristics, lifestyle factors, and genotype distribution in case and control groups}

The subjects included 161 cases and 483 controls: $45.3 \%$ of the 
subjects were female, and $54.7 \%$ were male. The medians of LDL-C in the case group were significantly higher than those in the control group $(p<0.05)$ (Table 1$)$. The $\chi^{2}$ test revealed significant differences in fruits/vegetables intake and the genotypes of rs10431036, rs11214109, rs11583680, rs17321515, and rs2954029 between the case group and the control group ( $p<$ $0.05)$. All of the studied SNPs in the control subjects were in HWE ( $p>0.05$ ) (Supplementary Table 1, only online).

\section{Associations of genetic models and lifestyle with the risk of IS}

The associations among $B C O 2$ and TRIB1 and lifestyle factors with IS were examined under each gene model. With or without adjustment for confounding factors of age, sex, waist circumference, smoking, drinking, LDL-C, the dominant models of four SNPs (rs10431036, rs11214109, rs17321515, and rs2954029), the additive models of two SNPs (rs17321515 and rs2954029), and fruits/vegetables intake were found to be significantly as-

Table 1. General Characteristics, Lifestyle Factors, and Genotype Distribution between Case and Control Groups

\begin{tabular}{|c|c|c|c|c|}
\hline Characteristics & Case (n=161) & Control ( $n=483)$ & $t / z / \chi^{2}$ & p value ${ }^{*}$ \\
\hline Age, median (IQR), yr & $66(19)$ & $66.5(16)$ & -0.236 & 0.813 \\
\hline Sex, $n(\%)$ & & & 0.000 & 0.999 \\
\hline Male & $88(55.1)$ & $264(55.1)$ & & \\
\hline Female & $73(44.9)$ & $219(44.9)$ & & \\
\hline Waist circumference, median (IQR), cm & $81(11)$ & $80(11)$ & -0.329 & 0.732 \\
\hline SBP, median (IOR), mm Hg & $139(30)$ & $140(26)$ & -1.538 & 0.124 \\
\hline DBP, median (IQR), mm Hg & $82(14)$ & $83(13)$ & -0.895 & 0.371 \\
\hline BMI, median (IQR), kg/m² & $22.98(3.85)$ & $23.18(4.07)$ & -1.056 & 0.291 \\
\hline TC, median (IQR), mmol/L & $4.93(1.54)$ & $4.95(1.26)$ & -0.164 & 0.870 \\
\hline TG, median (IOR), mmol/L & $1.34(0.87)$ & $1.33(0.89)$ & -0.109 & 0.913 \\
\hline HDL-C, median (IQR), mmol/L & $1.21(0.35)$ & $1.21(0.37)$ & -0.728 & 0.466 \\
\hline LDL-C, median (IQR),mmol/L & $3.165(1.43)$ & $2.94(1.15)$ & -2.477 & 0.013 \\
\hline Current smoking, $n(\%)$ & $35(20.2)$ & $106(21.2)$ & 0.073 & 0.787 \\
\hline Current alcohol intake, $\mathrm{n}(\%)$ & $43(24.9)$ & $148(29.6)$ & 1.423 & 0.233 \\
\hline Fruits/vegetables intake, $\mathrm{n}(\%)$ & & & 9.284 & 0.002 \\
\hline Inadequate & 24 (14.9) & $129(26.7)$ & & \\
\hline Adequate & $137(85.1)$ & $354(73.3)$ & & \\
\hline rs10431036 & & & 18.957 & $<0.001$ \\
\hline GG & $45(28.3)$ & $217(45.02)$ & & \\
\hline GA & $95(59.7)$ & 203 (42.12) & & \\
\hline$A A$ & $19(12)$ & $62(12.86)$ & & \\
\hline rs11214109 & & & 11.582 & 0.009 \\
\hline CC & $49(2.3)$ & $212(3.8)$ & & \\
\hline CT & $88(27.2)$ & $210(34.4)$ & & \\
\hline $\mathrm{TT}$ & $22(70.5)$ & $60(61.8)$ & & \\
\hline rs11583680 & & & 7.42 & 0.06 \\
\hline CC & $114(2.9)$ & $384(79.84)$ & & \\
\hline CT & 41 (29.5) & 93 (19.33) & & \\
\hline $\mathrm{TT}$ & 4 (67.3) & $4(0.83)$ & & \\
\hline rs17321515 & & & 14.642 & 0.002 \\
\hline GG & $31(19.3)$ & $162(33.60)$ & & \\
\hline GA & $90(60)$ & $236(48.97)$ & & \\
\hline AA & $38(23.6)$ & $84(17.43)$ & & \\
\hline rs2954029 & & & 13.071 & 0.004 \\
\hline $\mathrm{TT}$ & 33 (20.75) & 163 (33.82) & & \\
\hline TA & 88 (55.35) & 235 (48.76) & & \\
\hline AA & $38(23.9)$ & 84 (17.42) & & \\
\hline
\end{tabular}

IQR, interquartile range; SBP, systolic blood pressure; DBP, diastolic blood pressure; BMI, body mass index; TC, total cholesterol; TG, triglyceride; HDL-C, highdensity lipoprotein cholesterol; LDL-C, low-density lipoprotein cholesterol.

$p$ value $<0.05$ was considered statistically significant and maintained significance using the Benjamini-Hochberg procedure with the false discovery rate at 0.09 .

${ }^{*}$ Student's t-test, $\chi^{2}$ test and Wilcoxon's rank sum test. 
Table 2. Associations of Genetic Models and Lifestyles with Risk of Ischemic Stroke

\begin{tabular}{|c|c|c|c|c|c|}
\hline SNP/lifestyle & Genotype/Classification & Unadjusted OR (95\% CI) & Unadjusted $p$ value & Adjusted OR (95\% CI) & Adjusted $p$ value \\
\hline \multicolumn{6}{|l|}{ rs10431036 } \\
\hline \multirow{2}{*}{ Additive } & $\mathrm{GA} / \mathrm{GG}$ & $2.26(1.51-3.38)$ & $<0.001$ & $2.36(1.56-3.57)$ & $<0.001$ \\
\hline & $\mathrm{AA} / \mathrm{GG}$ & $1.48(0.81-2.71)$ & 0.207 & $1.61(0.87-2.99)$ & 0.131 \\
\hline Dominant & $\mathrm{GA}+\mathrm{AA} / \mathrm{GG}$ & $2.0(1.36-2.93)$ & $<0.001$ & $2.08(1.41-20.08)$ & $<0.001$ \\
\hline Recessive & $\mathrm{AA} / \mathrm{GA}+\mathrm{GG}$ & 1 (0.59-1.70) & 0.998 & $1.07(0.62-1.82)$ & 0.828 \\
\hline \multicolumn{6}{|l|}{ rs11214109 } \\
\hline \multirow{2}{*}{ Additive } & CT/CC & $1.81(1.22-2.70)$ & 0.003 & $1.86(1.24-2.79)$ & 0.003 \\
\hline & TT/CC & $1.59(0.89-2.83)$ & 0.118 & $1.59(0.88-2.87)$ & 0.124 \\
\hline Dominant & $\mathrm{CT}+\mathrm{TT} / \mathrm{CC}$ & $1.70(1.17-2.48)$ & 0.006 & $1.73(1.18-2.54)$ & 0.005 \\
\hline Recessive & $\mathrm{TT} / \mathrm{CT}+\mathrm{CC}$ & $1.21(0.73-2.02)$ & 0.460 & $1.20(0.72-2.02)$ & 0.484 \\
\hline \multicolumn{6}{|l|}{ rs17321515 } \\
\hline \multirow{2}{*}{ Additive } & GA/AA & $2.0(1.27-3.14)$ & 0.003 & $1.93(1.22-3.06)$ & 0.005 \\
\hline & GG/AA & $2.36(1.37-4.06)$ & 0.002 & $2.23(1.28-3.87)$ & 0.005 \\
\hline Dominant & $\mathrm{GA}+\mathrm{AA} / \mathrm{GG}$ & $1.98(1.29-3.03)$ & 0.002 & $1.94(1.26-2.99)$ & 0.003 \\
\hline Recessive & $\mathrm{AA} / \mathrm{GA}+\mathrm{AA}$ & $1.55(1.01-2.37)$ & 0.045 & $1.51(0.98-2.32)$ & 0.064 \\
\hline \multicolumn{6}{|l|}{ rs2954029 } \\
\hline \multirow{2}{*}{ Additive } & TA/AA & $1.85(1.18-2.89)$ & 0.007 & $1.79(1.14-2.82)$ & 0.012 \\
\hline & TT/AA & $2.23(1.31-3.81)$ & 0.003 & $2.10(1.20-3.63)$ & 0.008 \\
\hline Dominant & $\mathrm{TA}+\mathrm{AA} / \mathrm{TT}$ & $1.85(1.22-2.82)$ & 0.040 & $1.82(1.19-2.77)$ & 0.006 \\
\hline Recessive & $\mathrm{AA} / \mathrm{TA}+\mathrm{TT}$ & $1.55(1.01-2.37)$ & 0.045 & $1.51(0.98-2.33)$ & 0.064 \\
\hline \multicolumn{6}{|l|}{ rs11583680 } \\
\hline \multirow{2}{*}{ Additive } & $\mathrm{CT} / \mathrm{CC}$ & 1.49 (0.97-2.273 & 0.067 & $1.42(0.90-2.22)$ & 0.132 \\
\hline & TT/CC & 3.37 (0.83-13.68) & 0.089 & $3.84(0.30-16.87)$ & 0.075 \\
\hline Dominant & (CT+TT) / CC & 1.54 (1.02-2.33) & 0.038 & $1.49(0.96-2.31)$ & 0.074 \\
\hline Recessive & $\mathrm{TT} / \mathrm{CT}+\mathrm{CC}$ & 3.07 (0.98-9.68) & 0.055 & $2.79(0.83-9.42)$ & 0.097 \\
\hline Fruits/vegetables & Inadequate/adequate & $0.48(0.30-0.78)$ & 0.003 & $0.49(0.30-0.80)$ & 0.005 \\
\hline
\end{tabular}

$\mathrm{OR}$, odds ratio; $\mathrm{Cl}$, confidence interval.

Adjusted for age, sex, body mass index, waist, low-density lipoprotein cholesterol, smoking, drinking in logistic regression model. $p$ value $<0.05$ was considered statistically significant and maintained significance using the Benjamini-Hochberg procedure with the false discovery rate at 0.09 .

sociated with IS (Table 2). In dominant models, the GA+AA genotype of rs10431036, the CT+TT genotype of rs11214109, the GA+AA genotype of rs17321515, and the TA+AA genotype of rs2954029 increased the risk of IS (adjusted OR=2.08, 95\% CI = 1.41-20.08, $p<0,001$; adjusted $\mathrm{OR}=1.73,95 \% \mathrm{CI}=1.18-2.54, p=$ 0.005 ; adjusted $\mathrm{OR}=1.94,95 \% \mathrm{CI}=1.26-2.99, p=0.003$; adjusted $\mathrm{OR}=1.82,95 \% \mathrm{CI}=1.19-2.77, p=0.006)$. In additive models, the GG genotype of rs17321515 and the TT genotype of rs2954029 increased the risk of IS (adjusted OR=2.23, 95\% CI=1.28-3.87, $p=0.005$; adjusted $\mathrm{OR}=2.10,95 \% \mathrm{CI}=1.20-3.63, p=0.008)$. Adequate intake of fruits/vegetables posed lower susceptibility to IS than inadequate fruits/vegetables intake (adjusted $\mathrm{OR}=0.49$, 95\% CI $=0.30-0.80, p=0.005$ ).

\section{Interactions among $B C O 2$, TRIB1, lifestyle factors, and IS}

Table 3 show the effects of the interactions among four SNPs (rs10431036, rs11214109, rs17321515, and rs2954029) in two genes (BCO2 and TRIB1) and lifestyle factors on IS. The following results were adjusted for age, sex, waist circumference, BMI, smoking, drinking, and LDL-C. In rs10431036, compared with people who carried the GG genotype and had adequate intake of fruits/vegetables, those with the GA+AA genotype who had adequate/inadequate intake of fruits/vegetables and with the GG genotype who had inadequate fruits/vegetables were at a higher risk of IS (OR=5.84, 95\% CI=1.63-20.86, $p=$ 0.007; $\mathrm{OR}=9.33,95 \% \mathrm{CI}=2.82-30.86, p<0.001$; $\mathrm{OR}=5.12,95 \% \mathrm{CI}=$ 1.51-17.28, $p=0.009)$. In rs11214109, compared with people who carried the GG genotype and had adequate intake of fruits/vegetables, those with the CT+TT genotype who had adequate/ inadequate intake of fruits/vegetables and with the CC genotype who had inadequate fruits/vegetables were at a higher risk of IS ( $\mathrm{OR}=5.66,95 \% \mathrm{CI}=1.58-20.25, p=0.008$; $\mathrm{OR}=8.53$, 95\% CI=2.57-28.29, $p<0.001$; OR=5.74, 95\% CI=1.70-19.35, $p=$ 0.005). Compared with people who carried the GG genotype of rs17321515 and had adequate intake of fruits/vegetables, those with the GA+AA genotype who had adequate or inadequate intake of fruits/vegetables and those with the GG genotype who had inadequate fruits/vegetables were at a higher risk of IS ( $\mathrm{OR}=4.67,95 \% \mathrm{CI}=1.03-21.22, p=0.046$; $\mathrm{OR}=8.77$, 95\% CI=2.04-37.80, $p=0.004$; $\mathrm{OR}=4.81,95 \% \mathrm{CI}=1.08-21.40, p=$ 0.039). In rs2954029, compared with people who carried the 
Tian-Yu Zhao, et al.

Table 3. Interaction between Gene Polymorphism and Fruits/Vegetables Intake for the Risk of Ischemic Stroke

\begin{tabular}{|c|c|c|c|c|c|c|c|}
\hline \multirow{2}{*}{ Genotypes } & \multicolumn{2}{|c|}{$\begin{array}{l}\text { Adequate fruits/ } \\
\text { vegetables }\end{array}$} & \multicolumn{2}{|c|}{$\begin{array}{l}\text { Inadequate fruits/ } \\
\text { vegetables }\end{array}$} & \multirow{2}{*}{$\begin{array}{c}\text { OR }(95 \% \mathrm{Cl}) \\
\text { for inadequate } \\
\text { fruits/vegetables within } \\
\text { strata of genotype }\end{array}$} & \multirow{2}{*}{ RERI* (95\%CI) } & \multirow{2}{*}{$\begin{array}{c}p \\
\text { value }\end{array}$} \\
\hline & $\begin{array}{c}\text { Case/ } \\
\text { control (n) }\end{array}$ & OR (95\% Cl) & $\begin{array}{c}\text { Case/ } \\
\text { control (n) }\end{array}$ & OR (95\% Cl) & & & \\
\hline rs10431036 & & & & & & $-0.62(-5.19-3.94)$ & 0.789 \\
\hline \multirow[t]{3}{*}{ Non-risk allele carriers (GG) } & $3 / 56$ & & $44 / 162$ & & & & \\
\hline & & 1 & & $5.12(1.51-17.28)$ & $5.12(1.51-17.28)$ & & \\
\hline & & & & $p=0.009$ & $p=0.009$ & & \\
\hline \multirow[t]{3}{*}{ Risk allele carriers (GA+AA) } & $21 / 73$ & & $93 / 192$ & & & & \\
\hline & & $5.84(1.63-20.86)$ & & $9.33(2.82-30.86)$ & $1.52(0.86-2.70)$ & & \\
\hline & & $p=0.007$ & & $p<0.001$ & $p=0.151$ & & \\
\hline \multirow{2}{*}{$\begin{array}{l}\text { OR }(95 \% \mathrm{CI}) \text { for risk allele carriers within } \\
\text { strata of fruits/vegetables intake }\end{array}$} & & $5.84(1.63-20.86)$ & & $1.84(1.20-2.82)$ & & & \\
\hline & & $p=0.007$ & & $p=0.005$ & & & \\
\hline rs11214109 & & & & & & $-1.82(-7.05-3.39)$ & 0.493 \\
\hline \multirow[t]{3}{*}{ Non-risk allele carriers (CC) } & $3 / 55$ & & $48 / 158$ & & & & \\
\hline & & 1 & & $5.74(1.70-19.35)$ & $5.74(1.70-19.35)$ & & \\
\hline & & & & $p=0.005$ & $p=0.005$ & & \\
\hline \multirow[t]{3}{*}{ Risk allele carriers (CT+TT) } & $21 / 74$ & & $89 / 196$ & & & & \\
\hline & & $5.66(1.58-20.25)$ & & $8.53(2.57-28.29)$ & $1.51(0.99-2.29)$ & & \\
\hline & & $p=0.008$ & & $p<0.001$ & $p=0.055$ & & \\
\hline \multirow{2}{*}{$\begin{array}{l}\text { OR (95\% Cl) for risk allele carriers within } \\
\text { strata of fruits/vegetables intake }\end{array}$} & & $5.66(1.58-20.25)$ & & $1.46(0.82-2.60)$ & & & \\
\hline & & $p=0.008$ & & $p=0.200$ & & & \\
\hline rs17321515 & & & & & & $0.287(-3.49-4.06)$ & 0.881 \\
\hline \multirow[t]{3}{*}{ Non-risk allele carriers (GG) } & $2 / 38$ & & $31 / 125$ & & & & \\
\hline & & 1 & & $4.81(1.08-21.40)$ & $4.81(1.08-21.40)$ & & \\
\hline & & & & $p=0.039$ & $p=0.039$ & & \\
\hline \multirow[t]{3}{*}{ Risk allele carriers (GA+AA) } & $22 / 91$ & & $106 / 229$ & & & & \\
\hline & & $4.67(1.03-21.22)$ & & $8.77(2.04-37.80)$ & $1.79(1.03-3.09)$ & & \\
\hline & & $p=0.046$ & & $p=0.004$ & $p=0.036$ & & \\
\hline \multirow{2}{*}{$\begin{array}{l}\text { OR (95\% CI) for risk allele carriers within } \\
\text { strata of fruits/vegetables intake }\end{array}$} & & $4.67(1.03-21.22)$ & & $1.19(1.17-2.98)$ & & & \\
\hline & & $p=0.046$ & & $p=0.009$ & & & \\
\hline rs2954029 & & & & & & $-0.13(-4.10-3.82)$ & 0.950 \\
\hline \multirow[t]{3}{*}{ Non-risk allele carriers (TT) } & $2 / 38$ & & $33 / 126$ & & & & \\
\hline & & 1 & & $5.07(1.14-22.53)$ & $5.07(1.14-22.53)$ & & \\
\hline & & & & $p=0.033$ & $p=0.033$ & & \\
\hline \multirow[t]{3}{*}{ Risk allele carriers (TA+AA) } & $22 / 91$ & & $104 / 228$ & & & & \\
\hline & & $4.66(1.03-21.17)$ & & $8.61(1.99-37.11)$ & $1.78(1.03-3.07)$ & & \\
\hline & & $p=0.046$ & & $p=0.004$ & $p=0.04$ & & \\
\hline \multirow{2}{*}{$\begin{array}{l}\text { OR (95\% CI) for risk allele carriers within } \\
\text { strata of fruits/vegetables intake }\end{array}$} & & $4.66(1.03-21.17)$ & & $1.74(1.10-2.75)$ & & & \\
\hline & & $p=0.046$ & & $p=0.018$ & & & \\
\hline
\end{tabular}

$\mathrm{OR}$, odds ratio; $\mathrm{Cl}$, confidence interval; $\mathrm{RERI}$, relative excess risk due to interaction.

Adjusted for age, sex, waist circumference, body mass index, smoking, drinking, low-density lipoprotein cholesterol. $p$ value $<0.05$ was considered statistically significant and maintained significance using the Benjamini-Hochberg procedure with the false discovery rate at 0.09 .

${ }^{*}$ RERls were calculated according Knol, et al. ${ }^{19}$

TT genotype and had adequate intake of fruits/vegetables, those with the TA+AA genotype who had adequate/ inadequate intake of fruits/vegetables and those with the CC genotype who had inadequate fruits/vegetables were at a higher risk of IS (OR=4.66, 95\% CI=1.03-21.17, $p=0.046$; $\mathrm{OR}=8.61,95 \% \mathrm{CI}=$ 1.99-37.11, $p<0.004 ; \mathrm{OR}=5.07,95 \% \mathrm{CI}=1.14-22.53, p=0.033)$.

The interactions between gene polymorphism and fruits/ vegetables intake were not found in the additive model ( $p$ values of RERI >0.05).

\section{Associations for $B C O 2$ and TRIBI haplotypes with IS risk}

LD analysis for the SNPs showed obvious LD between two pairs of SNPs (Supplementary Table 2, only online). The hap- 
Table 4. Frequencies of Haplotypes among Cases and Controls and Association with Risk of Ischemic Stroke Haplotypes

\begin{tabular}{|c|c|c|c|c|c|}
\hline Haplotypes & Frequency & Case, n (\%) & Controls, n (\%) & OR (95\% Cl) & $p$ value \\
\hline \multicolumn{6}{|c|}{ rs10431036-rs11214109 } \\
\hline GC & 0.6241 & $174.55(54.9)$ & $624.89(64.8)$ & 1 & \\
\hline $\mathrm{AC}$ & 0.0148 & $11.45(3.6)$ & $9.11(0.9)$ & $6.30(2.34-16.95)$ & $<0.001$ \\
\hline AT & 0.3432 & $121.55(38.2)$ & $317.89(33.0)$ & $1.42(1.07-1.88)$ & 0.015 \\
\hline GT & 0.0180 & $10.45(3.3)$ & $12.11(1.3)$ & $3.11(1.28-7.56)$ & 0.012 \\
\hline \multicolumn{6}{|l|}{ rs2954029-rs17321515 } \\
\hline AA & 0.5530 & $152.00(47.8)$ & $560.00(58.1)$ & 1 & \\
\hline GT & 0.4446 & $164.00(51.6)$ & $403.00(41.8)$ & $1.52(1.16-1.99)$ & 0.002 \\
\hline Rare group* & 0.0024 & $1.93(0.6)$ & $1.00(0.1)$ & 10.36 (0.90-119.47) & 0.061 \\
\hline
\end{tabular}

$\mathrm{OR}$, odds ratio; $\mathrm{Cl}$, confidence interval.

Adjusted for age, sex, waist circumference, body mass index, smoking, drinking, low-density lipoprotein cholesterol. $p$ value $<0.05$ was considered statistically significant and maintained significance using the Benjamini-Hochberg procedure with the false discovery rate at 0.09 .

${ }^{*}$ Haplotypes with frequency $<0.01$ were pooled into the rare group.

lotype frequencies of the four SNPs were compared between IS cases and control subjects (Table 4). The haplotypes AC, AT, and GT (comprising rs10431036 and rs11214109) were found to be associated with an increased risk of $\mathrm{IS}(\mathrm{OR}=6.30,95 \% \mathrm{CI}=$ 2.34-16.95, $p<0.001 ; \mathrm{OR}=1.42,95 \% \mathrm{CI}=1.07-1.88, p=0.015$; OR= $3.11,95 \% \mathrm{CI}=1.28-7.56, p=0.012$ ). The haplotype GT (comprising rs2954029 and rs17321515) was also found to be associated with an increased risk of $\mathrm{IS}(\mathrm{OR}=1.52,95 \% \mathrm{CI}=1.16-1.99, p=$ 0.002).

\section{DISCUSSION}

Here, we investigated associations for $B C O 2, T R 1 B 1$, and PCSK9 polymorphisms with IS in Chinese Han people in southern China. The results showed that different genetic backgrounds and lifestyles would lead to different risks of IS.

TRIB1 is a gene that plays a structural role in the cholesterol metabolism and the atherosclerosis process. Our study showed that the GG genotype of rs17321515 and the TT genotype of rs2954029 in TRIB1 in dominant and addictive models posed a higher risk of IS. The association remained the same after adjustment for confounding factors. These results indicated that the G mutation site of rs17321515 and the T mutation site of rs2954029 were risk factors for IS, which was consistent with the study by Járomi, et al. ${ }^{20}$ rs2954029 is located on chromosome 8q24 and encodes the protein tribbles homolog 1, which has been shown to be associated with LDL-C, HDL-C, and TG concentrations, as well as the risk of CAD. ${ }^{21}$ Also, rs17321515 was discovered as a very compelling SNP effecting lipoprotein metabolism. ${ }^{14}$ The overexpression of TRIB1 significantly reduced the plasma levels of very low-density lipoprotein, LDLC, HDL-C, and TG, which may be a possible mechanism underlying the association between TRIBI and IS.

$B C O 2$ is a potential candidate gene that plays a substantial role in the inner membrane of mitochondria through regulation of electron transport chain complexes. Our study showed that the GG genotype of rs10431036 and the CC genotype of rs11214109 of BCO2 in a dominant model posed a higher risk of IS. The association remained after adjustment for confounding factors. These were newly identified SNPs for susceptibility to IS. No studies on $\mathrm{BCO} 2$ and IS have been reported. Nevertheless, genetic studies have linked $\mathrm{BCO} 2$ mutations to obesity in humans, ${ }^{22,23}$ and obese people are thought to be more susceptible to IS, suggesting that $\mathrm{BCO} 2$ might influence IS in obese individuals.

This study also analyzed associations between fruits/vegetables intake and IS. We found that adequate fruits/vegetables intake could reduce the risk of IS, which was consistent with most previous studies. ${ }^{15-17}$ Fruits/vegetables can effectively protect the cardio-cerebrovascular system from damage, while lacking fruits/vegetables was found to result in up to twice the incidence of cardio-cerebrovascular events. ${ }^{15,16}$ This association can be explained by several biological mechanisms. An adequate intake of fruits/vegetables lowers blood pressure and improves microvascular function. ${ }^{24}$ It also has favorable effects on other cardiovascular risk factors, such as plasma levels of TC and LDL-C, BMI, waist circumference, and inflammation, thereby reducing the risk of IS. In addition, adequate intake of fruits and vegetables increases micronutrients, carbohydrates, and fiber levels and potentially reduces fat intake and the risk of IS. ${ }^{17}$

In case-control studies, RERI is generally considered the standard measure of additive model interactions. Our study investigated the interactions of gene-lifestyle factors with the risk of IS. People who ate inadequate fruits/vegetables or carried risk genotypes had a higher risk of IS. The risk of IS increased when two risk factors were present together, compared to either of them alone, though the interaction index of four SNPs and fruits/vegetables intake was not statistically significant. In addition, we found that the haplotypes AC, AT, and GT, composed of rs10431036 and rs11214109, and the haplotype GT, composed of rs2954029 and rs17321515, increased the risk of IS.

This study had some limitations. First, the study might lead to unstable research results, owing to the small sample size, which makes our observations highly susceptible to type 2 er- 
rors. Second, community-based cohorts involving self-reported data might suffer from an uncertainty concerning the validity of the data. Third, a change of exposure to environmental risk factors during the follow-up period were difficult to assess. Finally, the research conclusions might only be applicable to people in southern China because of geographical restrictions. To confirm our present findings, randomized, large-scale, longterm studies from different regions and ethnic or social background will be needed.

In conclusion, polymorphisms of two SNPs (rs10431036 and rs11214109) in BCO2, two SNPs (rs17321515 and rs2954029) in TRIB1, and fruits/vegetables intake were found to be associated with IS in people in southern China. These results provide the theoretical basis for gene screening to prevent cerebrovascular diseases.

\section{ACKNOWLEDGEMENTS}

This work was supported by the Program for Zhejiang Leading Team of Science and Technology Innovation (no. 2011R 50021).

We thank everyone who participated in the present study.

\section{AUTHOR CONTRIBUTIONS}

Tian-Yu Zhao, Zheng Li, and Lei Yang had the original idea for the study and, with all co-authors, carried out the design. Song Lei was responsible for recruitment and follow-up of study participants. TianYu Zhao drafted the manuscript, which was revised by all authors. Liu Huang and Song Lei provided advanced statistical methods. All authors read and approved the final manuscript.

\section{ORCID iDs}

$\begin{array}{ll}\text { Tian-Yu Zhao } & \text { https://orcid.org/0000-0002-4424-7959 } \\ \text { Zheng Li } & \text { https://orcid.org/0000-0002-7335-6599 } \\ \text { Song Lei } & \text { https://orcid.org/0000-0001-9517-1088 } \\ \text { Liu Huang } & \text { https://orcid.org/0000-0002-0827-7210 } \\ \text { Lei Yang } & \text { https://orcid.org/0000-0001-7618-9936 }\end{array}$

\section{REFERENCES}

1. GBD 2016 Stroke Collaborators. Global, regional, and national burden of stroke, 1990-2016: a systematic analysis for the Global Burden of Disease Study 2016. Lancet Neurol 2019;18:439-58.

2. Bravata DM, Daggy J, Brosch J, Sico JJ, Baye F, Myers LJ, et al. Comparison of risk factor control in the year after discharge for ischemic stroke versus acute myocardial infarction. Stroke 2018;49: 296-303.

3. Wei LK, Au A, Menon S, Gan SH, Griffiths LR. Clinical relevance of MTHFR, eNOS, ACE, and ApoE gene polymorphisms and serum vitamin profile among Malay patients with ischemic stroke. J Stroke Cerebrovasc Dis 2015;24:2017-25.

4. Venkat P, Shen Y, Chopp M, Chen J. Cell-based and pharmacological neurorestorative therapies for ischemic stroke. Neuropharmacology 2018;134(Pt B):310-22.

5. Chen X, Zhou L, Zhang Y, Yi D, Liu L, Rao W, et al. Risk factors of stroke in Western and Asian countries: a systematic review and meta-analysis of prospective cohort studies. BMC Public Health 2014;14:776.

6. Juan J, Jiang X, Tang X, Wu Y, Sun K, Xiang X, et al. Joint effects of PON1 polymorphisms and vegetable intake on ischemic stroke: a family-based case control study. Int J Mol Sci 2017;18:2652.

7. Olsson S, Jood K, Melander O, Sjögren M, Norrving B, Nilsson M, et al. Lack of association between genetic variations in the KALRN region and ischemic stroke. Clin Biochem 2011;44:1018-20.

8. Yue YH, Bai XD, Zhang HJ, Li YM, Hu L, Liu LY, et al. Gene polymorphisms affect the effectiveness of atorvastatin in treating ischemic stroke patients. Cell Physiol Biochem 2016;39:630-8.

9. Yang Y, Wu W, Wang L, Ding Y. Lack of association between interleukin-1 receptor antagonist gene 86-bp VNTR polymorphism and ischemic stroke: a meta-analysis. Medicine (Baltimore) 2018;97: e11750.

10. Ikeoka T, Hayashida N, Nakazato M, Sekita T, Murata-Mori F, Ando $\mathrm{T}$, et al. The $\mathrm{A}>\mathrm{T}$ polymorphism of the tribbles homolog 1 gene is associated with serum triglyceride concentrations in Japanese community-dwelling women. Tohoku J Exp Med 2014;233:149-53.

11. Schmidt AF, Swerdlow DI, Holmes MV, Patel RS, Fairhurst-Hunter $\mathrm{Z}$, Lyall DM, et al. PCSK9 genetic variants and risk of type 2 diabetes: a mendelian randomisation study. Lancet Diabetes Endocrinol 2017;5:97-105.

12. Zhang L, Song K, Zhu M, Shi J, Zhang H, Xu L, et al. Proprotein convertase subtilisin/kexin type 9 (PCSK9) in lipid metabolism, atherosclerosis and ischemic stroke. Int J Neurosci 2016;126:67580.

13. Lim JY, Liu C, Hu KQ, Smith DE, Wang XD. Ablation of carotenoid cleavage enzymes (BCO1 and $\mathrm{BCO} 2$ ) induced hepatic steatosis by altering the farnesoid $\mathrm{X}$ receptor/miR-34a/sirtuin 1 pathway. Arch Biochem Biophys 2018;654:1-9.

14. Aung LH, Yin RX, Wu DF, Li Q, Yan TT, Wang YM, et al. Association of the TRIB1 tribbles homolog 1 gene rs17321515 A>G polymorphism and serum lipid levels in the Mulao and Han populations. Lipids Health Dis 2011;10:230.

15. Micha R, Shulkin ML, Peñalvo JL, Khatibzadeh S, Singh GM, Rao M, et al. Etiologic effects and optimal intakes of foods and nutrients for risk of cardiovascular diseases and diabetes: systematic reviews and meta-analyses from the Nutrition and Chronic Diseases Expert Group (NutriCoDE). PLoS One 2017;12:e0175149.

16. Keller HH, Carrier N, Slaughter S, Lengyel C, Steele CM, Duizer L, et al. Making the Most of Mealtimes (M3): protocol of a multi-centre cross-sectional study of food intake and its determinants in older adults living in long term care homes. BMC Geriatr 2017;17:15.

17. Guzek D, Głąbska D, Lange E, Jezewska-Zychowicz M. A Polish study on the influence of food neophobia in children (10-12 years old) on the intake of vegetables and fruits. Nutrients 2017;9:563.

18. Society of Neurology, Chinese Medical Association. Guidelines for the diagnosis and treatment of acute ischemic stroke in China in 2014. Chin J Neurol 2015;48:246-57.

19. Knol MJ, VanderWeele TJ. Recommendations for presenting analyses of effect modification and interaction. Int J Epidemiol 2012;41:514-20.

20. Járomi L, Csöngei V, Polgár N, Rappai G, Szolnoki Z, Maász A, et al. Triglyceride level-influencing functional variants of the ANGPTL3, CILP2, and TRIB1 loci in ischemic stroke. Neuromolecular Med 2011;13:179-86.

21. Douvris A, Soubeyrand S, Naing T, Martinuk A, Nikpay M, Williams A, et al. Functional analysis of the TRIB1 associated locus linked to plasma triglycerides and coronary artery disease. J Am Heart Assoc 2014;3:e000884.

22. Brocato J, Hernandez M, Laulicht F, Sun H, Shamy M, Alghamdi 
MA, et al. In vivo exposures to particulate matter collected from Saudi Arabia or nickel chloride display similar dysregulation of metabolic syndrome genes. J Toxicol Environ Health A 2015;78: 1421-36.

23. Wu L, Guo X, Wang W, Medeiros DM, Clarke SL, Lucas EA, et al. Molecular aspects of $\beta, \beta$-carotene-9', 10'-oxygenase 2 in carot- enoid metabolism and diseases. Exp Biol Med (Maywood) 2016; 241:1879-87.

24. Hu D, Huang J, Wang Y, Zhang D, Qu Y. Fruits and vegetables consumption and risk of stroke: a meta-analysis of prospective cohort studies. Stroke 2014;45:1613-9. 